\title{
Effect of quintessence on holographic fermionic spectrum
}

\author{
Xiao-Mei Kuang ${ }^{1,2, a}$, Jian-Pin Wu $\mathbf{W u}^{3, b}$ \\ ${ }^{1}$ Center for Gravitation and Cosmology, College of Physical Science and Technology, Yangzhou University, Yangzhou 225009, China \\ ${ }^{2}$ Instituto de Física, Pontificia Universidad Católica de Valparaíso, Casilla, 4059 Valparaiso, Chile \\ ${ }^{3}$ Institute of Gravitation and Cosmology, Department of Physics, School of Mathematics and Physics, Bohai University, Jinzhou 121013, China
}

Received: 12 July 2017 / Accepted: 14 September 2017 / Published online: 9 October 2017

(c) The Author(s) 2017. This article is an open access publication

\begin{abstract}
In this letter, we investigate the holographic fermionic spectrum without/with dipole coupling dual to the Reissner-Nordström anti-de Sitter (RN-AdS) black brane surrounded by quintessence. We find that the low energy excitation of this fermionic system without dipole coupling behaves as a non-Fermi liquid. In particular, the introduction of quintessence aggravates the degree of deviation from a Fermi liquid. For the system with dipole coupling, the phase transition from (non-)Fermi liquid to Mott phase can be observed. The ratio between the width of gap and the critical temperature, beyond which the gap closes, is also worked out. We find that this ratio is larger than that of the holographic fermionic system dual to the RN-AdS black brane and even the material of $\mathrm{VO}_{2}$. It means that our holographic system with quintessence can model new phenomena of the condensed matter system and provide some new insights in their regard.
\end{abstract}

\section{Introduction}

In order to understand the exotic phenomenas in strongly coupled matters, such as high temperature superconductors and the heavy fermions, topics of anti-de Sitter/condensed matter theory (AdS/CMT), as an application of gauge/gravity duality [1-4], are flourishing and lead to remarkable progress.

Holographic fermions belong to one of the important implements, which couples fermion fields to a $U(1)$ gauge field in the gravitational action in the bulk theory proposed in [5]. Then the authors of [6-8] treated the fermions as a probe and study the spectral function dual to Reissner-Nordström anti-de Sitter (RN-AdS) black brane. Their results show that a Fermi surface emerges and the low energy excitations near the Fermi surface exhibit a marginal Fermi liquid, or (non)Fermi liquid behaviors, which is controlled by the conformal

\footnotetext{
a e-mail: xmeikuang@gmail.com

b e-mail: jianpinwu@mail.bnu.edu.cn
}

dimensions in the IR conformal field theory (CFT) dual to $\mathrm{AdS}_{2}$ geometry. So it is expected that one may provide significant insight disclosing the basic principle hidden behind the strangle metal phase. The extended study of the holographic fermionic system dual to more general bulk theory has been explored in [9-25]. Later, a dipole coupling term between the massless charged fermions and the gauge field was introduced in [26,27], which brings about a Mott insulating phase and the spectral weight transfers between bands in the dual boundary theory. This indicates that the doped Mott phase is mimicked in the holographic framework. The proposal has inspired further study of the dipole coupling effect on the holographic fermionic systems [28-38]. Moreover, the fermionic spectral function without translational symmetry has been disclosed in [39-43].

Observational evidence shows that our universe is undergoing an accelerative expanding, driven by dark energy which is still unknown. Usually, dark energy is studied via its footprints on the expansion of the universe and on the growth of the large scale structure. From the recent data of the cosmic microwave background (CMB), supernova and baryon acoustic oscillations, the cosmological models with a dark energy fluid with the state parameter $W$ close to -1 are competitive because it shows the current Hubble expansion accelerates. Besides, kinds of model of dark energy have been proposed, such as the cosmological constant model [44], quintessence model [45,46], phantom model [47], k-essence model [48] and so on. Later, by treating quintessence as an unknown fluid, Kiselev studied Einstein's field equation surrounded by quintessential dark matter and obtained a new solution dependent on the state parameter $W$ of the quintessence in [49]. The quasinormal modes and Hawking radiation of the quintessence black branes have been investigated in [50,51], which confirmed that quintessence is significant to the black hole and modifies the quasinormal frequencies as well as the standard results of Hawking radiation. 
Recently, Chen et al. extended the black hole solutions of [49] into a planar quintessence AdS black brane, and they studied the effect of quintessence on the s-wave and p-wave holographic superconductor via gauge/gravity duality $[52,53]$. It was found that the presence of dark energy has effects on the condensation and brings about richer physics for the holographic superconductor. The aim of this paper is to study the holographic fermionic spectrum dual to the four dimensional quintessence AdS black brane. To this end, we construct the charged planar RN-AdS black brane surrounded by quintessence in four dimensional theory. Moreover, in order to study the influences of the quintessence on the systems with more significant physical phases from holography, especially, the Mott system, which is typical in strong coupling physics, we will introduce a dipole interaction term between the fermions and gauge field proposed in [26] into the bulk action. We firstly disclose how the existence of quintessence affects the Fermi surface including the Fermi momentum and the dispersion relation. Then we study the emergence of a gap due to the strong dipole coupling and the low energy excitation in both zero temperature and finite temperature. We also analyze the influence of quintessence on all these properties of the fermionic spectrum. The study is very significant because it will help us to further understand the connections among AdS black holes, strongly coupled sectors and quintessence matter.

The remaining of this paper is organized as follows. We will, in parallel, construct the charged quintessence AdS black brane and study the UV and IR behavior of the solution in Sect. 2. In Sect. 3, we will analyze the Dirac equation and the Green function of the operator dual to the Dirac fields. We numerically solve the deformed Dirac equation; then we investigate the fermionic spectral function without and with dipole coupling in Sect. 4 and in Sect. 5, respectively. Then we turn on the temperature and study the Mott gap closing due to the finite temperature in Sect. 6. The last section is for the conclusion and discussion.

\section{The charged RN-AdS black brane surrounded by quintessence}

We consider the Einstein-Maxwell action with negative cosmological constant in four dimensions,

$S=\int \mathrm{d} x^{4} \sqrt{-g}\left(R+\frac{6}{L^{2}}-\frac{1}{4} F_{\mu \nu} F^{\mu \nu}\right)$

where the field strength for the Maxwell field is $F_{\mu \nu}=$ $\partial_{\mu} A_{\nu}-\partial_{\nu} A_{\mu}$. The action gives us the equations of motion as

$$
\begin{aligned}
& \nabla_{\nu} F^{\mu \nu}=0, \\
& R_{\mu \nu}-\frac{1}{2} R g_{\mu \nu}-3 g_{\mu \nu}=\frac{1}{2} T_{\mu \nu}^{(M)},
\end{aligned}
$$

where we have set the AdS radius $L=1$ and the energy momentum tensor of the Maxwell field is $T_{\mu \nu}^{(M)}=F_{\mu \rho} F_{\nu}^{\rho}-$ $\frac{1}{4} g_{\mu \nu} F^{\rho \sigma} F_{\rho \sigma}$.

In order to construct the RN-AdS black brane surrounded by quintessence, we follow [49] to consider the quintessence field as the perfect fluid, and we add its energy momentum tensor,

$T_{t}^{t}=T_{r}^{r}=-\rho, \quad T_{x}^{x}=T_{y}^{y}=\frac{\rho(3 W+1)}{2}$,

to the right hand side of the Einstein equation (3). Here $W$ is the state parameter with the range

$-1<W<-\frac{1}{3}$

meant to bring about the acceleration of our universe, and $W=-1$ describes the cosmological constant term.

Considering the ansatz $A_{\mu}=(\phi(r), 0,0,0)$ and the metric

$$
\begin{aligned}
\mathrm{d} s^{2} & =-g_{t t} \mathrm{~d} t^{2}+g_{r r} \mathrm{~d} r^{2}+g_{x x} \mathrm{~d} x^{2}+g_{y y} \mathrm{~d} y^{2} \\
& =-f(r) \mathrm{d} t^{2}+\frac{\mathrm{d} r^{2}}{f(r)}+r^{2}\left(\mathrm{~d} x^{2}+\mathrm{d} y^{2}\right) .
\end{aligned}
$$

The general solution to the fields equations of motions is

$$
\begin{aligned}
& f(r)=r^{2}-\frac{M}{r}+\frac{\mu^{2}}{4 r^{2}}-\frac{a}{r^{3 W+1}}, \\
& \phi(r)=\mu\left(1-\frac{r_{h}}{r}\right),
\end{aligned}
$$

where $r_{h}$ is the event horizon satisfying $f\left(r_{h}\right)=0 . M$ is the mass of the black brane,

$M=r_{h}^{3}+\frac{\mu^{2}}{4 r_{h}}-\frac{a}{r_{h}^{3 W}}$,

and $a$ is a positive integral constant which is to give us a positive energy for quintessence, $\rho=-\frac{a}{2} \frac{3 W}{r^{3}(W+1)}$. Note that the solution recovers the $\mathrm{RN}-\mathrm{AdS}$ black hole when the integral constant is $a=0$ or the energy of quintessence is $\rho=0$. The temperature and the entropy of the black brane can be calculated as

$$
\begin{aligned}
T & =\frac{f^{\prime}\left(r_{h}\right)}{4 \pi}=\frac{3}{4 \pi}\left(1-\frac{\mu^{2}}{12 r_{h}^{4}}+\frac{a W}{r_{h}^{3(W+1)}}\right), \\
S & =\int_{0}^{r_{h}} \frac{1}{T}\left(\frac{\partial M}{\partial r_{h}}\right) \mathrm{d} r_{h}=\pi r_{h}^{2} .
\end{aligned}
$$


To simplify, we do the following rescaling:

$$
\begin{aligned}
r & \rightarrow r_{h} r, \quad t \rightarrow \frac{t}{r_{h}}, \quad(x, y) \rightarrow \frac{1}{r_{h}}(x, y), \\
T & \rightarrow \frac{T}{r_{h}}, \quad \phi \rightarrow r_{h}^{2} \phi,
\end{aligned}
$$

and so we can set $r_{h}=1$. Then we rewrite the metric factor $f(r)$ and the gauge fields $\phi$ as follows:

$$
\begin{aligned}
& f=r^{2}-\frac{1}{r}+\frac{\mu^{2}(1-r)}{4 r^{2}}-\frac{a}{r^{3 W+1}}\left(1-r^{3 W}\right), \\
& \phi=\mu\left(1-\frac{1}{r}\right),
\end{aligned}
$$

and the dimensionless temperature in the form

$T=\frac{3}{4 \pi}\left[1-\frac{\mu^{2}}{12}+a W\right]$.

It is obvious that the condition for the zero-temperature limit is $\mu=\sqrt{12(1+a W)}$ Then at zero temperature and in the $r \rightarrow r_{h}=1$ limit, we can reduce the case to

$$
\begin{aligned}
\left.f(r)\right|_{T=0, r \rightarrow 1} & \simeq\left[6+\frac{3}{2} a W(1-3 W)\right](r-1)^{2} \\
& \equiv \frac{1}{\tilde{L}^{2}}(r-1)^{2} .
\end{aligned}
$$

Therefore, at the zero temperature, we obtain the near horizon geometry $\mathrm{AdS}_{2} \times \mathbb{R}^{2}$ with the curvature radius $\tilde{L} \equiv$ $1 / \sqrt{6+\frac{3}{2} a W(1-3 W)}$ of $\mathrm{AdS}_{2}$ which depends explicitly on the state parameter of the quintessence. So, near the horizon, under the transformation $r-1=\epsilon \frac{\tilde{L}^{2}}{\varsigma}$ and $t=\epsilon^{-1} \tau$, the metric and the gauge fields are derived in the limit $\epsilon \rightarrow 0$ with finite $\varsigma$ and $\tau$,

$\mathrm{d} s^{2}=\frac{\tilde{L}^{2}}{\varsigma^{2}}\left(-\mathrm{d} \tau^{2}+\mathrm{d} \varsigma^{2}\right)+\mathrm{d} x^{2}+\mathrm{d} y^{2}, \quad \phi=\frac{\mu \tilde{L}^{2}}{\varsigma}$.

\section{The Dirac equation and Green function}

In this section, we construct the holographic fermionic model and derive the correlation functions.

\subsection{The Dirac equation}

We consider the Dirac action

$S_{D}=i \int \mathrm{d}^{4} x \sqrt{-g} \bar{\zeta}\left(\Gamma^{a} \mathcal{D}_{a}-m-i p \not{F}\right) \zeta$,

with a dipole moment coupling between the fermion and the gauge field. Here $\mathcal{D}_{a}=\partial_{a}+\frac{1}{4}\left(\omega_{\mu \nu}\right)_{a} \Gamma^{\mu \nu}-i q A_{a}$ and $\boldsymbol{F}=\frac{1}{2} \Gamma^{\mu \nu}\left(e_{\mu}\right)^{a}\left(e_{\nu}\right)^{b} F_{a b}$ with $\Gamma^{\mu \nu}=\frac{1}{2}\left[\Gamma^{\mu}, \Gamma^{\nu}\right]$ and the spin connection is $\left(\omega_{\mu \nu}\right)_{a}=\left(e_{\mu}\right)^{b} \nabla_{a}\left(e_{\nu}\right)_{b}$.
Starting from the above action, with the new definition $\zeta=$ $\left(-g g^{r r}\right)^{-\frac{1}{4}} \mathcal{F}$ and a Fourier transformation $\mathcal{F}=F e^{-i \omega t+i k x}$, we calculate the Dirac equation in the Fourier space as

$$
\begin{gathered}
\left(\sqrt{g^{r r}} \Gamma^{r} \partial_{r}-m\right) F-i(\omega+q \phi) \sqrt{g^{t t}} \Gamma^{t} F \\
+i\left(k \sqrt{g^{x x}}-p \sqrt{g^{t t} g^{r r}} \partial_{r} \phi\right) \Gamma^{x} F=0 .
\end{gathered}
$$

Note that we have set $k_{x}=k$ and $k_{y}=0$ because of the rotational symmetry in the $x-y$ plane. After we choose the usual gamma matrices as

$$
\begin{array}{ll}
\Gamma^{r}=\left(\begin{array}{cc}
-\sigma^{3} & 0 \\
0 & -\sigma^{3}
\end{array}\right), \quad \Gamma^{t}=\left(\begin{array}{cc}
i \sigma^{1} & 0 \\
0 & i \sigma^{1}
\end{array}\right), \\
\Gamma^{x}=\left(\begin{array}{cc}
-\sigma^{2} & 0 \\
0 & \sigma^{2}
\end{array}\right), & \cdots
\end{array}
$$

The Dirac equation has the form

$$
\begin{aligned}
& {\left[\left(\partial_{r}+m \sqrt{g_{r r}} \sigma^{3}\right)-\sqrt{\frac{g_{r r}}{g_{t t}}}(\omega+q \phi) i \sigma^{2}\right.} \\
& \left.-\left((-1)^{I} k \sqrt{\frac{g_{r r}}{g_{x x}}}-p \sqrt{g^{t t}} \partial_{r} \phi\right) \sigma^{1}\right] F_{I}=0
\end{aligned}
$$

with $I=1,2$. Decomposing $F_{I}$ into $F_{I}=\left(\mathcal{A}_{I}, \mathcal{B}_{I}\right)^{T}$, we find that the components obey the equations of motions,

$$
\begin{aligned}
& \left(\partial_{r}+m \sqrt{g_{r r}}\right) \mathcal{A}_{I}-\sqrt{\frac{g_{r r}}{g_{t t}}}(\omega+q \phi) \mathcal{B}_{I} \\
& -\left((-1)^{I} k \sqrt{\frac{g_{r r}}{g_{x x}}}-p \sqrt{g^{t t}} \partial_{r} \phi\right) \mathcal{B}_{I}=0, \\
& \left(\partial_{r}-m \sqrt{g_{r r}}\right) \mathcal{B}_{I}+\sqrt{\frac{g_{r r}}{g_{t t}}}(\omega+q \phi) \mathcal{A}_{I} \\
& -\left((-1)^{I} k \sqrt{\frac{g_{r r}}{g_{x x}}}-p \sqrt{g^{t t}} \partial_{r} \phi\right) \mathcal{A}_{I}=0 .
\end{aligned}
$$

Then defining the new field variables $\xi_{I} \equiv \frac{\mathcal{A}_{I}}{\mathcal{B}_{I}}$, we see that $\xi_{I}$ satisfies the flow equation,

$$
\begin{gathered}
\left(\partial_{r}+2 m \sqrt{g_{r r}}\right) \xi_{I}-\left(v_{-}+(-1)^{I} k \sqrt{\frac{g_{r r}}{g_{x x}}}\right) \\
-\left(v_{+}-(-1)^{I} k \sqrt{\frac{g_{r r}}{g_{x x}}}\right) \xi_{I}^{2}=0,
\end{gathered}
$$

where the $v_{ \pm}$are expressed as $v_{ \pm}=\sqrt{\frac{g_{g r}}{g_{t t}}}(\omega+q \phi) \pm$ $p \sqrt{g^{t t}} \partial_{r} \phi$.

\subsection{Green's function}

\subsubsection{UV limit}

In the UV limit $r \rightarrow \infty$, the Dirac equation (21) becomes

$$
\left[\partial_{r}+\frac{m}{r} \sigma^{3}-\frac{\omega+q \mu}{r^{2}} i \sigma^{2}-\left((-1)^{I} \frac{k}{r^{2}}-\frac{p \mu}{r^{3}}\right) \sigma^{1}\right] F_{I}=0,
$$


which can be further reduced in the limit of $r \rightarrow \infty$ to

$$
\left(\partial_{r}+\frac{m}{r} \sigma^{3}\right) F_{I} \approx 0 \text {. }
$$

The solution satisfying the above equation is

$F_{I} \stackrel{r \rightarrow \infty}{\approx} b_{I} r^{m}\left(\begin{array}{l}0 \\ 1\end{array}\right)+a_{I} r^{-m}\left(\begin{array}{l}1 \\ 0\end{array}\right)$,

which is not affected by the quintessence.

According to the discussion of Faulkner et al. [8], we can choose either $a_{I}$ or $b_{I}$ as the source when we quantize the Dirac field with different boundary conditions. In this work, we will choose $b_{I}$ as the source and $a_{I}$ as the response. Thus, in the regime of a linear response, the boundary Green functions can be extracted by $G_{I}=\frac{a_{I}}{b_{I}}$. Since we have the definition

$\xi_{I} \equiv \frac{\mathcal{A}_{I}}{\mathcal{B}_{I}}$

the boundary Green functions can be expressed in terms of $\xi_{I}$

$G(\omega, k)=\left(\begin{array}{cc}G_{1} & 0 \\ 0 & G_{2}\end{array}\right)=\lim _{r \rightarrow \infty} r^{2 m}\left(\begin{array}{cc}\xi_{1} & 0 \\ 0 & \xi_{2}\end{array}\right)$.

Also, from Eq. (24), we can see that the Green function has the following symmetry:

$G_{1}(\omega, k ; m, p)=G_{2}(\omega,-k ; m, p)$.

\subsubsection{IR limit}

The low frequency behavior of the Green function is determined by the near horizon geometry. In the IR limit, the near horizon geometry is $\mathrm{AdS}_{2} \times \mathbb{R}^{2}$ (see Eq. (17)), so the Dirac equation in the limit of $\omega \rightarrow 0$ is reduced to

$\varsigma \partial_{\varsigma} F_{I}-\left[m \tilde{L} \sigma^{3}+\left(p \mu-(-1)^{I} k\right) \tilde{L} \sigma^{1}-i \sigma^{2} q \mu \tilde{L}^{2}\right] F_{I}=0$.

Here we have chosen the same Gamma matrices (20) but change $\Gamma^{\varsigma}=-\Gamma^{r}$ to reflect the orientation between the coordinates $r$ and $\zeta$. As discussed in Ref. [8], the above equation is nothing but that for spinor fields with the following masses in an $\mathrm{AdS}_{2}$ background:

$\left[m, \tilde{m}_{I}=p \mu-(-1)^{I} k\right]$

with the time-reversal violating mass terms $\tilde{m}_{I}(I=1,2)$. The field $F_{I}^{(0)}(\varsigma)$ is dual to the spinor operators $\mathbb{O}_{I}$ in the IR $C F T_{1}$ theory where the retarded Green functions of $\mathbb{O}_{I}$ are with

$\nu_{I}(k)=\sqrt{\left(m^{2}+\tilde{m}_{I}^{2}\right) \tilde{L}^{2}-\left(q \mu \tilde{L}^{2}\right)^{2}} \quad(I=1,2)$,

and the conformal dimensions of the operators are $\delta_{I}=$ $v_{I}(k)+\frac{1}{2}$.

And then, by the matching method [8], the UV Green function of the fermionic field can be expressed in terms of the IR one as

$G_{I}=\frac{a_{I}}{b_{I}}=\frac{\left[a_{I}^{(0)}+\omega a_{I}^{(1)}+\cdots\right]+\left[\tilde{a}_{I}^{(0)}+\omega \tilde{a}_{I}^{(1)}+\cdots\right] \mathcal{G}_{I}(k, \omega)}{\left[b_{I}^{(0)}+\omega b_{I}^{(1)}+\cdots\right]+\left[\tilde{b}_{I}^{(0)}+\omega \tilde{b}_{I}^{(1)}+\cdots\right] \mathcal{G}_{I}(k, \omega)}$,

where the coefficients $a_{I}^{(n)}, \tilde{a}_{I}^{(n)}, b_{I}^{(n)}$ and $\tilde{b}_{I}^{(n)}$ are to be determined by numerically solving the bulk Dirac equation (24). Note that the above expression of the Green function is valid only if $2 v_{I}(k)$ is not an integer. For the case of $2 v_{I}(k)$ being an integer, the terms like $\omega^{n} \log (\omega)$ should be added. For detailed discussions, we can refer to [8]. Our remarks on the properties of the fermionic Green function are as follows.

- $v(k)$ is pure imaginary when the momentum $k$ falls into the range

$$
k \in \mathfrak{I}_{I}=\left[(-1)^{I} p \mu-q \mu \tilde{L},(-1)^{I} p \mu+q \mu \tilde{L}\right] .
$$

So when the Fermi momentum $k_{\mathrm{F}}$ belongs to this region, the peak of the Green function will lose its meaning as a Fermi surface and this region of momentum space is defined as the oscillatory region [8].

We can read off some features of the range (36). For minimal coupling with $p=0$, the oscillatory regions for the two-dimensional dual operator $G_{1}$ and $G_{2}$ coincide, i.e., $\mathfrak{I}_{1}=\mathfrak{I}_{2}=[-q \mu \tilde{L}, q \mu \tilde{L}]$. However, turning on the dipole coupling will make $\mathfrak{I}_{1}$ and $\mathfrak{I}_{2}$ separate with part of overlap. Further increasing $|p|$ until $|p|>q \tilde{L}$, the two oscillatory regions will lose the intersection. The features of the regimes $\mathfrak{I}_{1}$ and $\mathfrak{I}_{2}$ described above are shown in Fig. 1 where the orange region denotes $\mathfrak{I}_{2}$ for $G_{2}(\omega, k)$, while the blue region corresponds to $\mathfrak{I}_{1}$ for $G_{1}(\omega, k)$. Another feature we can see from Eq. (36) and Fig. 1 is that the two regions are symmetric according to $p=0$. Moreover, comparing the plots, we can observe that for larger state parameters, the boundary of the oscillatory region has a smaller slope.

$\mathcal{G}_{I}(k, \omega)=\left\{e^{-i \pi v_{I}(k)} \frac{\Gamma\left(-2 v_{I}(k)\right) \Gamma\left(1+v_{I}(k)-i q \mu \tilde{L}^{2}\right)\left[\left(m+i \tilde{m}_{I}\right) \tilde{L}-i q \mu \tilde{L}^{2}-v_{I}(k)\right]}{\Gamma\left(2 v_{I}(k)\right) \Gamma\left(1-v_{I}(k)-i q \mu \tilde{L}^{2}\right)\left[\left(m+i \tilde{m}_{I}\right) \tilde{L}-i q \mu \tilde{L}^{2}+v_{I}(k)\right]}\right\} \omega^{2 v_{I}(k)}$, 

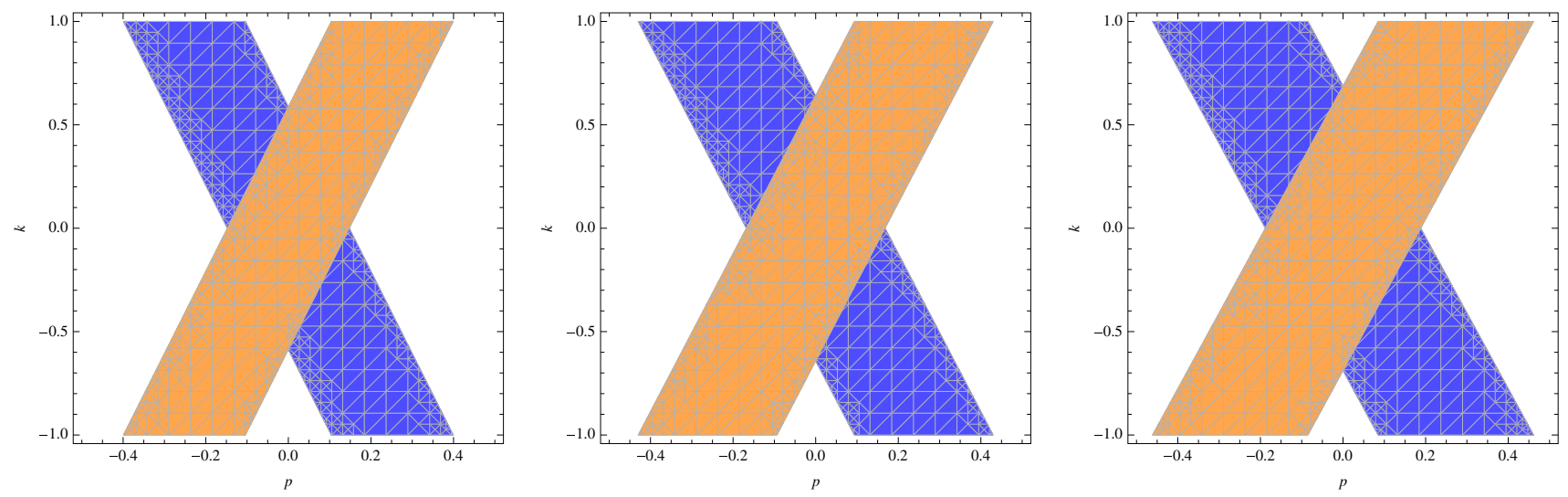

Fig. 1 The oscillatory regions. From left to right, the state parameters $W$ are $-0.95,-0.65,-0.35$. The orange region denotes the oscillatory region $\mathfrak{I}_{2}$ for $G_{2}(\omega, k)$ while the blue region corresponds to oscillatory region $\mathfrak{I}_{1}$ for $G_{1}(\omega, k)$. In the plots, we set the integral constant $a=1$

- Real $v_{I}(k)$ will help us to define the dispersion relation in the dual theory [8]

$$
\tilde{\omega}(\tilde{k}) \propto \tilde{k}^{\delta}, \quad \text { with } \delta= \begin{cases}\frac{1}{2 v_{I}\left(k_{\mathrm{F}}\right)}, & v_{I}\left(k_{\mathrm{F}}\right)<\frac{1}{2} \\ 1 & v_{I}\left(k_{\mathrm{F}}\right)>\frac{1}{2}\end{cases}
$$

which is a very important scaling and property of the Green function. Note that here $\tilde{k}=k-k_{\mathrm{F}}$ and $\tilde{\omega}$ is the real part of the frequency shift from zero. Using (37), once the Fermi momentum $k_{\mathrm{F}}$ is determined, we can directly compute the exponent of the dispersion relation $\delta$.

\section{Fermionic spectral without dipole coupling}

Now we shall turn to a study of the fermionic spectrum by numerically solving the bulk Dirac equations (24). To this end, we impose the in-going boundary condition at the horizon $r_{h}=1$, which is

$$
\left\{\begin{array}{l}
\xi_{I} \stackrel{r \rightarrow 1}{=} i \text { for } \omega \neq 0, \\
\xi_{I} \stackrel{r \rightarrow 1}{=} \frac{m \tilde{L}-v_{I}(k)}{q \mu \tilde{L}^{2}+\tilde{m}_{I} \tilde{L}} \text { for } \omega=0 .
\end{array}\right.
$$

For definiteness, we work only with the massless fermion, $m=0$, and set $q=0.5$ in what follows. Note that, as a test, we find that with state parameter $-1<W<-\frac{1}{3}$, the peak of the Green function is seriously suppressed by the positive constant $a$ in the black brane solution. In order to study the effect of state parameter on the fermionic spectrum of the dual boundary theory, we will set small $a=0.01$ if it is not specifically stated in the following studies.

In this section, we turn off the dipole coupling and mainly study the effect of the quintessence on the Green function. In particular, we shall focus on the Fermi momentum $k_{\mathrm{F}}$ where the Green function behaves as a peak at zero frequency, and we compute the exponent of the dispersion relation via Eq. (37).

The leftmost plots in Fig. 2 (plot in the upper panel is $3 \mathrm{D}$, while that in the bottom panel is the density) show a sharp peak at zero frequency and $k \simeq 0.92$, which is the Fermi momentum, for $a=0$ (RN black hole). While the middle and the rightmost plots in Fig. 2 exhibit the 3D and density plots for a fixed $a=0.01$ and $W=-0.35$ as well as $W=-0.95$, respectively. We see that with quintessence, there is also a peak for the Green function, but it is suppressed by the state parameters. The Fermi momentum increases as the state parameter becomes larger, which can be explicitly seen from Fig. 3. For $a=0.01$, in the allowed range $-1<$ $W<-\frac{1}{3}$, the momentum of the Fermi surface never enters in the oscillatory region marked in blue in the figure and it is always valid as a Fermi momentum. The exponent of the dispersion relation $\delta$ is over than 1 , which means the lower energy excitation near the Fermi surface behaves as nonFermi liquid because for Fermi liquid, it is always 1. While, for bigger $a$, see Fig. 4 with $a=1$, the Fermi momentum will enter the oscillatory region at a critical $W$, so $k_{\mathrm{F}}$ for smaller $W$ loses its meaning of Fermi momentum and $v_{2}\left(k_{\mathrm{F}}\right)$ becomes imaginary. For meaningful Fermi momentum, the related $\delta$ becomes smaller as $W$ increases, as shown in the right plot of Fig. 4, but still the corresponding lower energy excitation is a non-Fermi liquid. Comparing Figs. 3 and 4, the behavior of the lower energy excitation near the Fermi surface shifts more away from fermion liquid for larger $a$ because the dispersion relation is further away from unity.

We may understand this observation as follows. As revealed in $[6,8]$, the degree of deviation from a Fermi liquid becomes heavy as $q$ decreases. It can be interpreted as reminiscent of asymptotic freedom in high-density QCD if we assumed that the Fermi momentum $k_{\mathrm{F}}$ increases as $q$ increases. Note that the relevant quantity is the effective chemical potential $\mu_{q}=\mu q=g_{\mathrm{F}} q Q[6,8]$. When $W$ 

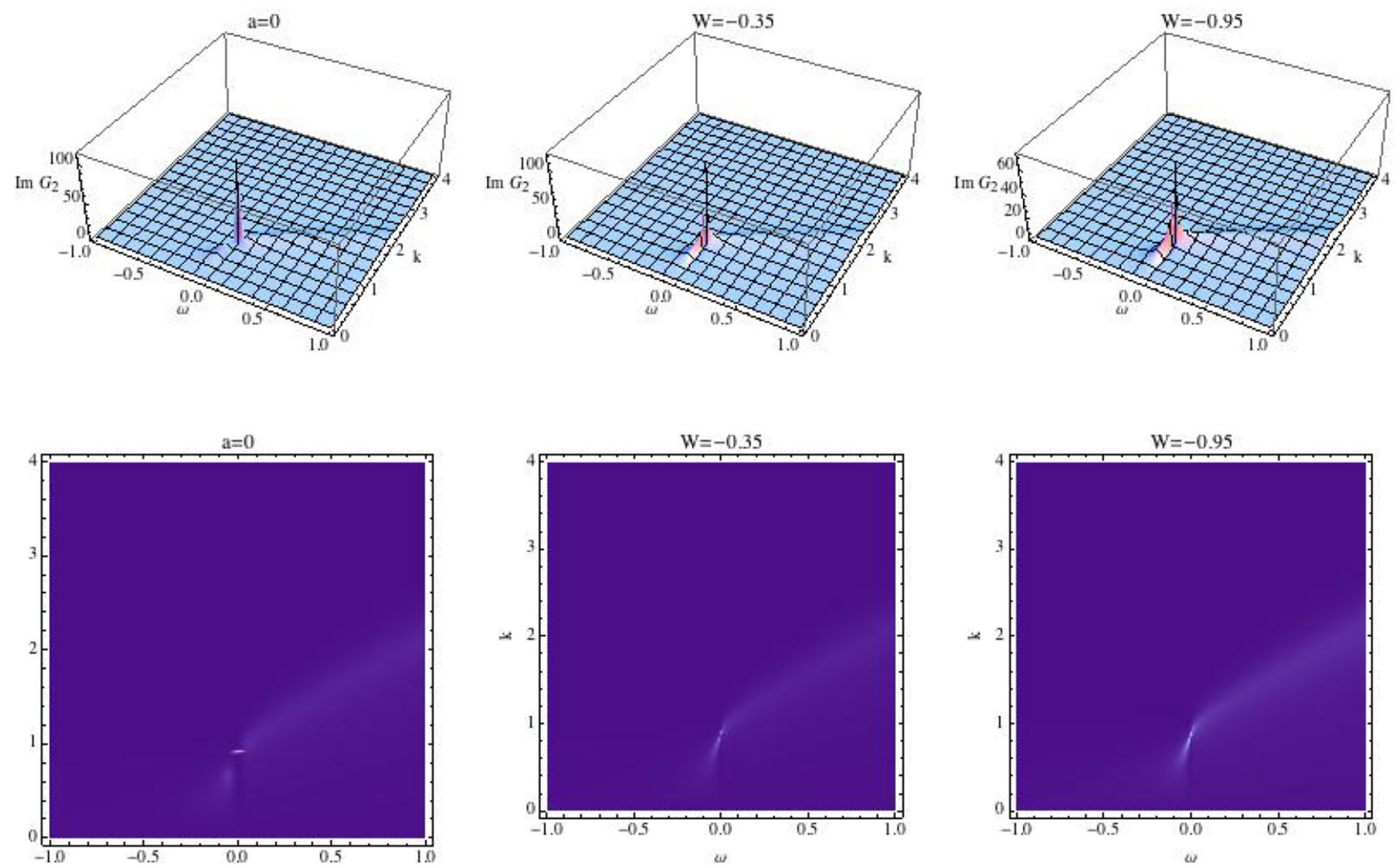

Fig. 2 Green function $G_{2}$
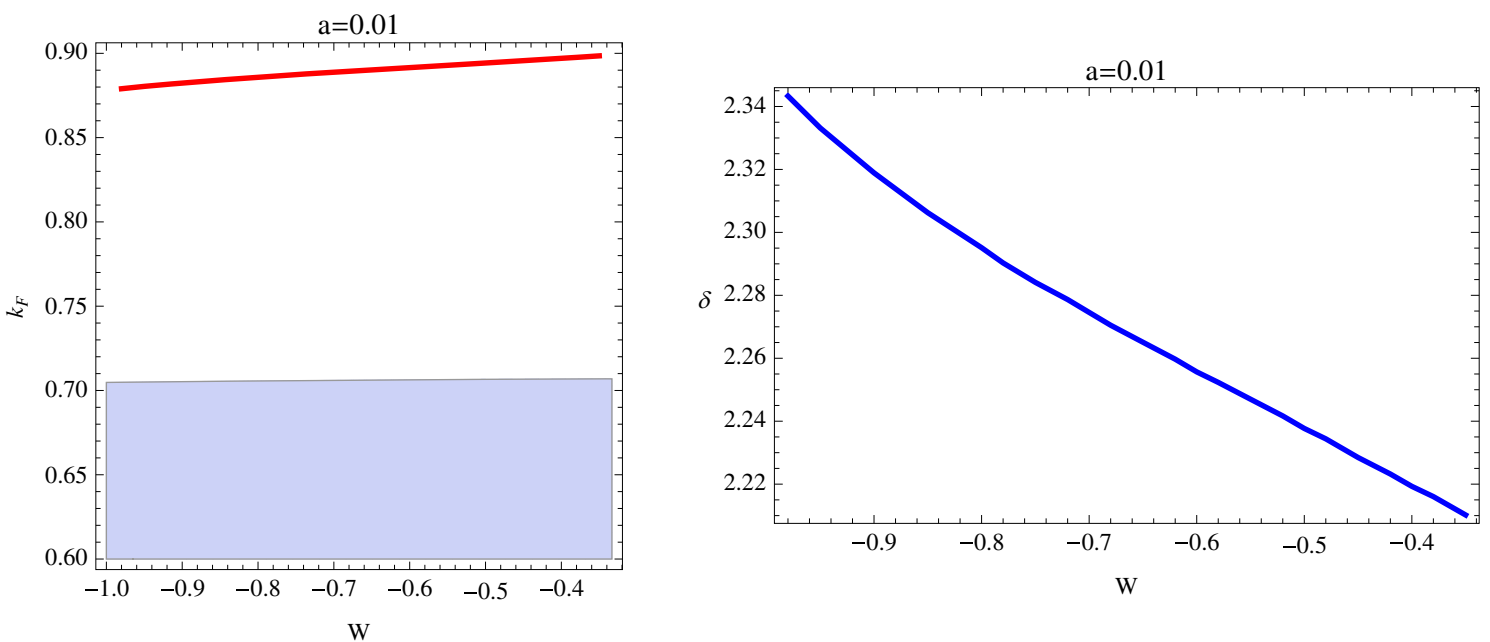

Fig. 3 Fermi momentum and the dispersion relation for $a=0.01$

is negative, which originates from the negative pressure of quintessence dark energy model, the zero-temperature chemical potential $\mu=\sqrt{12(1+a W)}$ becomes smaller for positive $a$ and so the effective potential is smaller for fixed $q$ and $g_{\mathrm{F}}$. Thus quintessence aggravates the degree of deviation from a Fermi liquid. The deeper physical mechanisms, why the introduction of quintessence makes the holographic system "more non-Fermi", are interesting and deserve further studying.

\section{Fermionic spectrum with dipole coupling}

In [26], a dipole coupling term between gauge field and Dirac field is introduced over the RN-AdS black hole to model the 


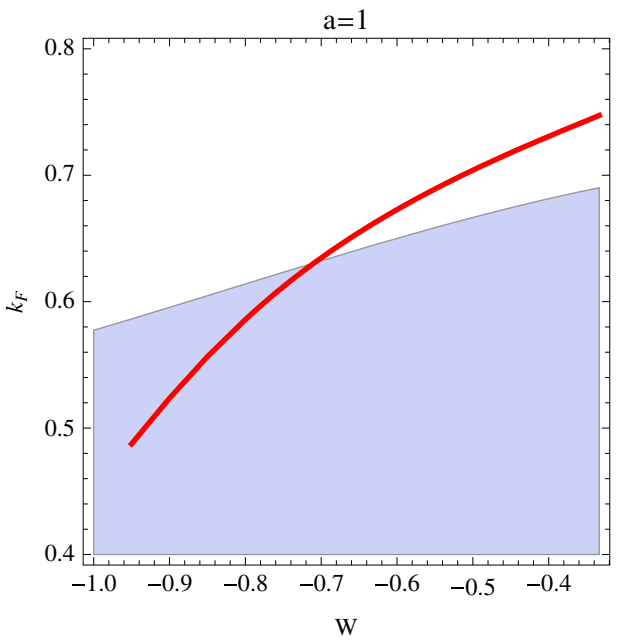

Fig. 4 Fermi momentum and the dispersion relation for $a=1$

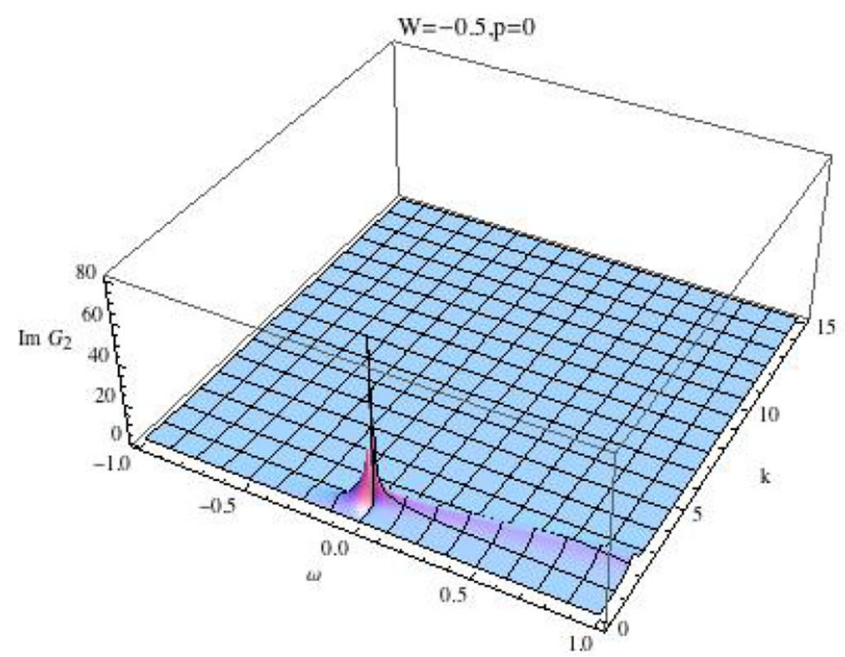

Fig. 5 Green function for $p=0$ (left) and $p=3$ (right)

generation of the Mott gap. One finds that with the increase of the dipole coupling parameter $p$, the holographic fermionic system undergoes a phase transition from (non-)Fermi liquid to Mott phase. Here, we shall study how the quintessence matter affects this phase transition and the properties of the (non-)Fermi liquid phase and Mott phase. We first focus on the zero-temperature case and generalize the study the finite temperature in next section.

\subsection{The emergence of the Mott gap}

In Fig. 5, we show the spectral function with fixed $W=-0.5$. For vanishing dipole coupling in the left plot, there is a peak at zero frequency denoting a Fermi surface, while in the right plot with $p=3$, a Mott gap around zero frequency replaces the peak. This phenomenon has been observed in [26] for the
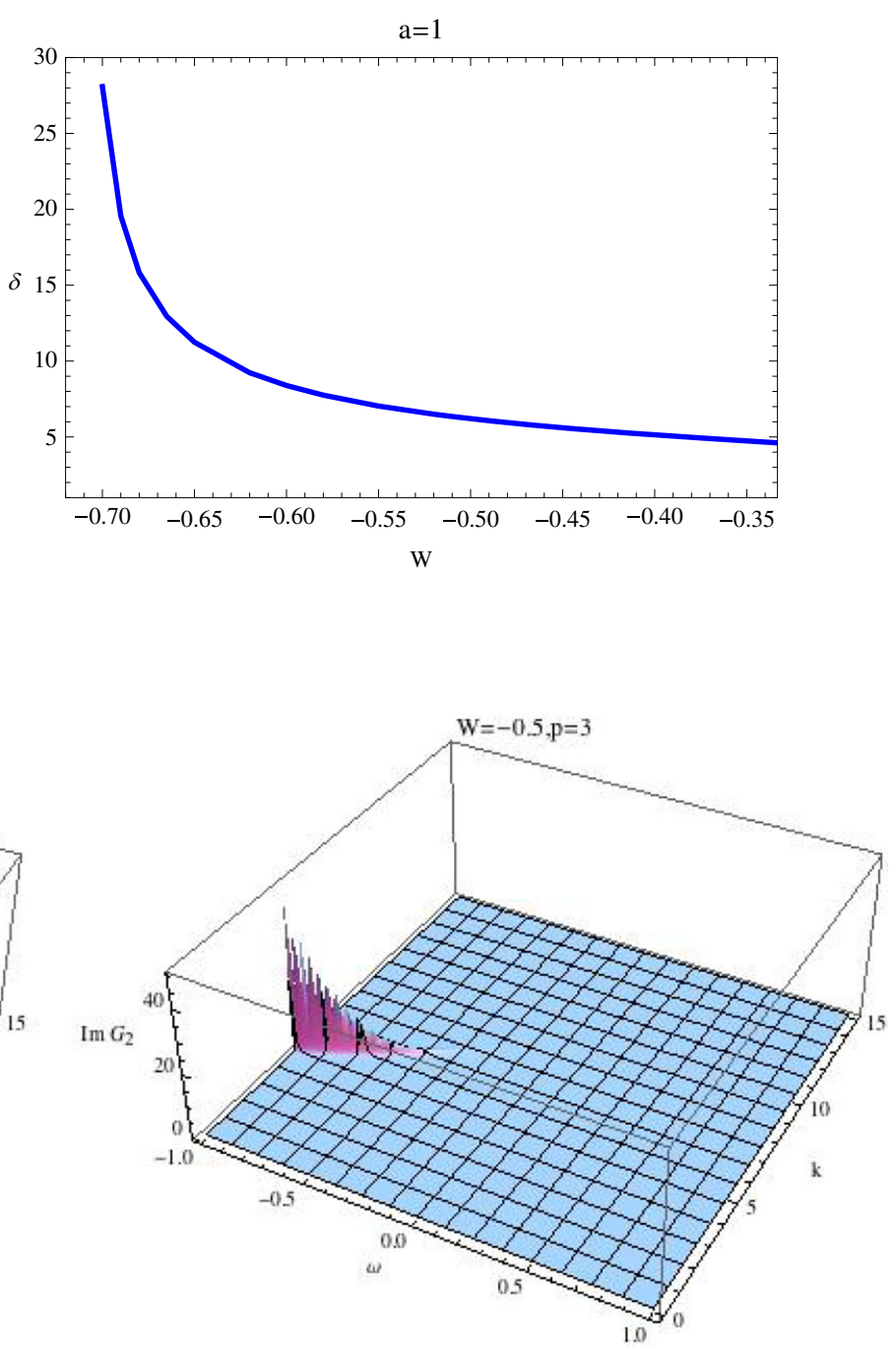

RN-AdS black hole. It is obvious that quintessence matter would not destroy the property.

To quantitatively study the process of emerging gap, we calculate the density of states $A(\omega)$ by integrating the fermionic spectral function $A(\omega ; k)=\operatorname{Tr}[\operatorname{ImG}(\omega ; \mathrm{k})]$ over $k$. The behavior of the density of states with different $p$ is shown in Fig. 6 where we fix $W=-0.5 .^{1}$ It is obvious that as the dipole coupling become stronger, the $A(\omega)$ is suppressed near the zero frequency. Finally a gap will open at critical value $p_{c}=2.602^{2}$, and further increasing the dipole coupling, the gap becomes wider. Meanwhile, in the process, the density of states for small $p$ is larger at positive frequency than at negative frequency, however, the behavior becomes

${ }^{1}$ For other state parameters, the behaviors are similar, we do not repeat the plots here.

${ }^{2}$ We define a gap when the spectral function is below $\sim 10^{-4}$ in this work. 


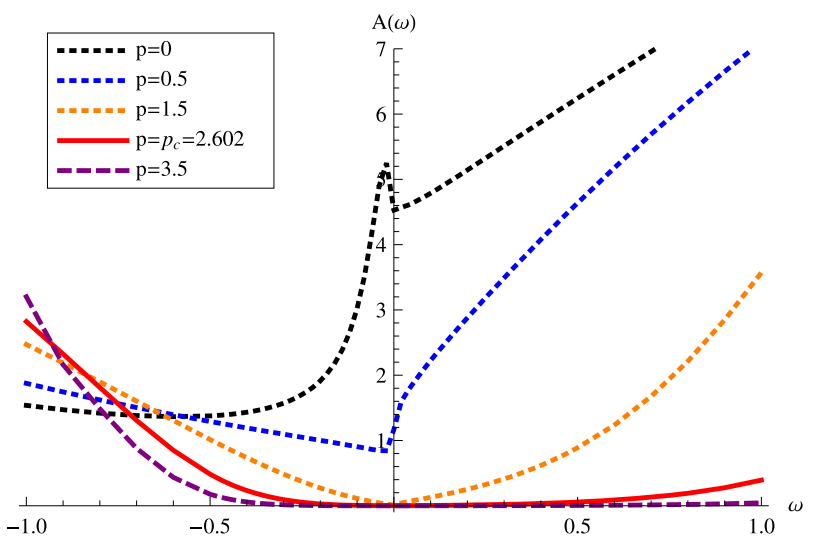

Fig. 6 The state density. We set $W=-0.5$

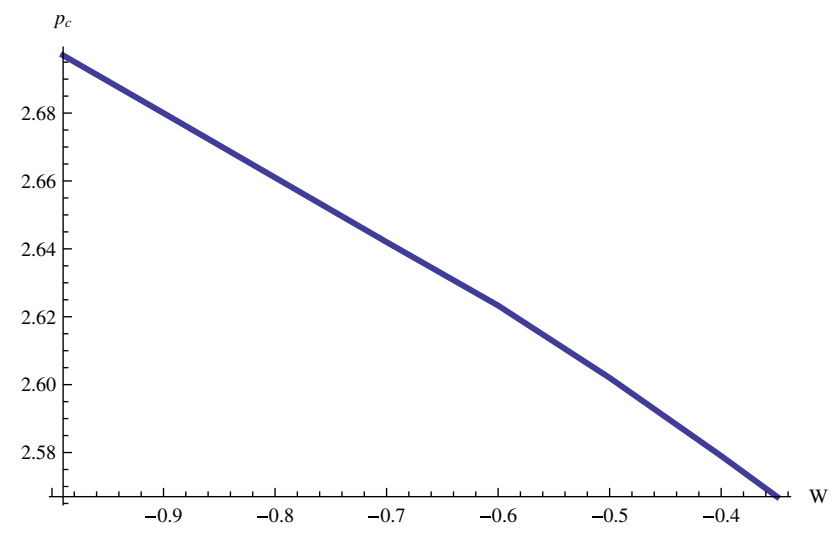

Fig. 7 The critical $p_{c}$ for opening a gap

converse for large $p$, which means the weight transfers from a positive frequency band to a negative one.

In order to study the influence of the state parameter, we find the critical dipole coupling for different $W$ which is shown in Fig. 7. We see that as the state parameter becomes larger, $p_{c}$ decreases, which means that the gap is easier to generate dynamically.

\subsection{The formation of the Fermi surface and the type of low} energy excitations

Now we turn to the case with $p<p_{c}$. Before the Mott gap forms, there is still a Fermi-like peak in the spectral function $[26,27]$. We know from Sect. 2 that oscillating region will make the Fermi momentum lose its meaning of denoting a Fermi surface. So here we intend to study the effect of the dipole coupling on the Fermi surface and the low energy excitation as well as its type in quintessence black brane.

In Table 1, we summarize some results of Fermi momenta. For fixed dipole coupling, large state parameter corresponds large Fermi momentum, which is consistent with the observation in Fig. 3. Also for fixed state parameters, the Fermi momentum increases as the dipole coupling becomes larger. Then we have to check if all the Fermi momenta listed in Table 1 are valid. Recalling the relation of (37) to compute the dispersion relation, we list our results of the exponents of dispersion relation in Table 2. Results for $p=0$ here match well with that found in Fig. 3. However, for smaller $p$, we find that the exponents of the dispersion relation is 1 , which means the excitation behaves as Fermi liquid. As $p$ increases, there is a transition from Fermi liquid to non-Fermi liquid with $\delta \neq 1$. Further increasing $p$, the Fermi momentum leads to imaginary $v_{2}(k)$, so the peak is not physical. From Table 2, we see that, for smaller state parameter, the Fermi momentum enters the oscillating region easier, i.e., it happens at smaller dipole coupling. This is reasonable because for smaller $W$, the border of the oscillation is steeper, as shown in Fig. 1, so that the Fermi momentum growing from the left can touch the border more easily.

\section{Finite temperature}

It was pointed out based on holography in [26,27] that beyond a certain finite temperature, the Mott gap closes and a metallic phase is recovered, which mimics the transition from insulating to metallic phase such as some Mott insulators disclosed $[54,55]$. Here we will study this phenomenon of the boundary theory dual to the black brane with quintessence.

In Fig. 8, with fixed $W=-0.65$ and $p=6$, we plot the Green function for $T=0$ and $T=0.15$, respectively. In the left plot for $T=0$, there exists a Mott gap which we also know from last section, while in the right plot with $T=0.15$, we see that the Mott gap is shaded, meaning the vanishing of the Mott insulator. The feature is consistent with that observed in other gravitational theories [27,29-31,33]. To describe how the gap is destroyed, we study the density of state at different temperatures, which is shown in Fig. 9. It is obvious that $A(\omega)$ around zero frequency is enhanced when the temperature is increased, and finally the gap closes.

Then we quantitatively study the ratio $\Delta / T_{\star}$ for $p=6$, where $\Delta$ and $T_{\star}$ denote the width of gap at zero temperature and the critical temperature at which the gap closes. The ratio affected by the state parameters are summarized in Table 3. As the state parameter increases, the ratio decreases slowly, but they are close to the value probed in $\mathrm{VO}_{2}$ where $\Delta / T_{\star} \simeq 20$ [54]. Note that the ratio found in RN-AdS black brane is $\Delta / T_{\star} \simeq 10$ [27]. So our holographic model with quintessence dark energy model is significant for understanding this transitional phenomenon found in Mott insulators.

\section{Conclusions and discussion}

Dark energy has been introduced to describe the accelerating expansion of the current universe. As one of the dark energy models, quintessence is an ordinary scalar field mini- 
Table 1 The Fermi momentum with dipole coupling for the different state parameters

Table 2 The dispersion relation $\delta$ with dipole coupling for the different state parameters

\begin{tabular}{llllllll}
\hline$p$ & \multicolumn{1}{l}{-0.1} & 0 & 0.05 & 0.1 & 0.11 \\
\hline-0.95 & -0.3548 & 0.1739 & 0.7169 & 0.8804 & 0.9672 & 1.0574 & 1.0758 \\
-0.65 & -0.3456 & 0.1816 & 0.7252 & 0.8902 & 0.9782 & 1.0699 & 1.0886 \\
-0.35 & -0.3433 & 0.1841 & 0.7307 & 0.8985 & 0.9885 & 1.0827 & 1.1020 \\
\hline
\end{tabular}

\begin{tabular}{|c|c|c|c|c|c|c|c|}
\hline \multicolumn{8}{|l|}{$p$} \\
\hline$W$ & -1 & -0.5 & -0.1 & 0 & 0.05 & 0.1 & 0.11 \\
\hline-0.95 & 1 & 1 & 1.5442 & 2.3318 & 3.3742 & 14.0310 & - \\
\hline-0.65 & 1 & 1 & 1.5222 & 2.2651 & 3.1802 & 7.8914 & 33.4263 \\
\hline-0.35 & 1 & 1 & 1.5079 & 2.2102 & 3.0192 & 5.9717 & 8.7205 \\
\hline
\end{tabular}
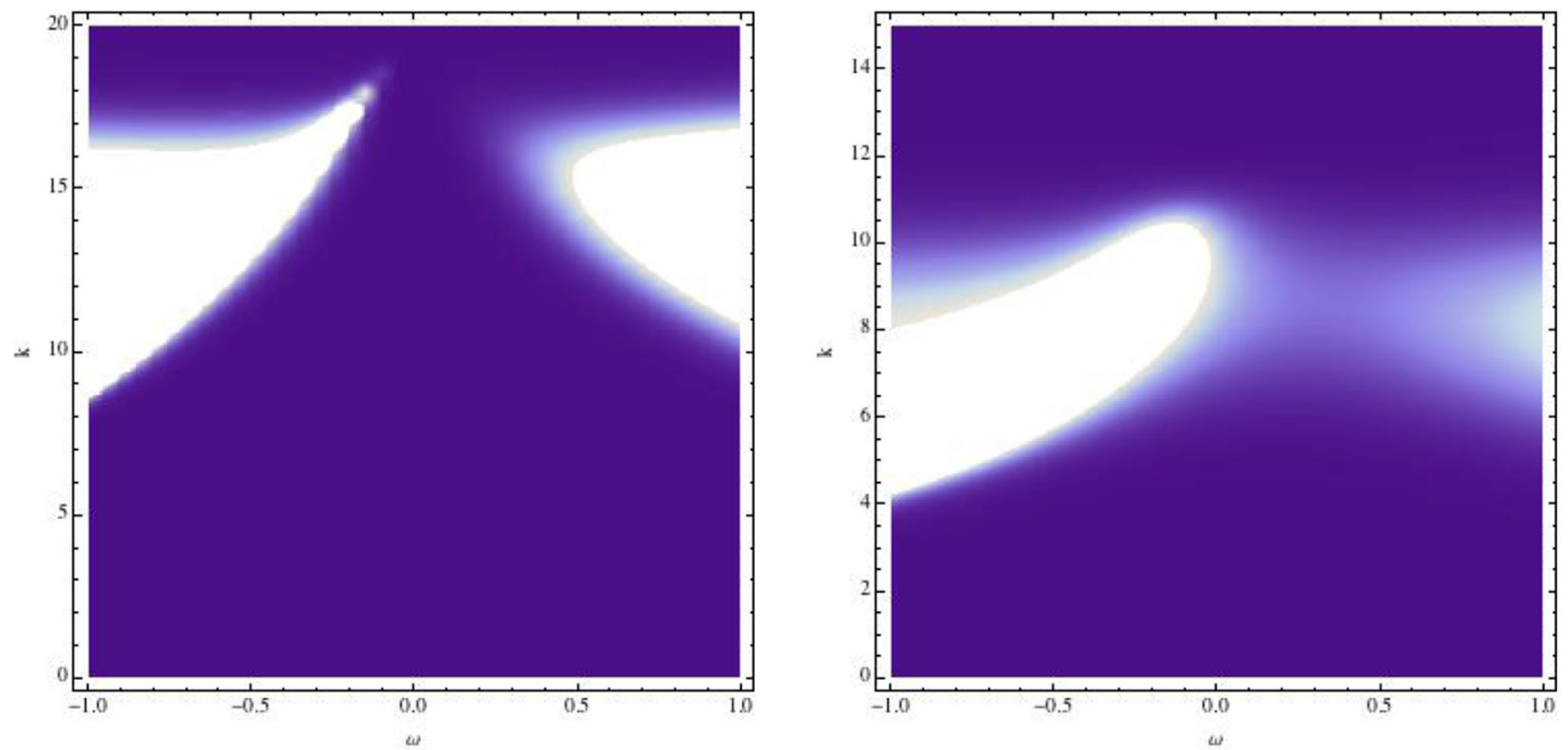

Fig. 8 Fermionic Green function for $T=0$ and $T=0.15$. We set $p=6$ and $W=-0.65$

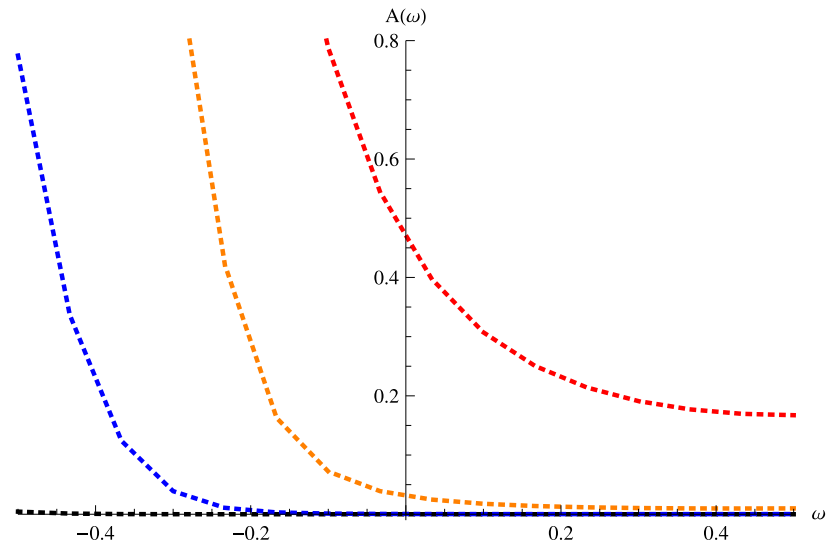

Fig. 9 Density of state for different temperatures with $p=6$ and $W=$ -0.65 . From upper to lower, the temperatures are $0.22,0.18,0.12,0$ mally coupled to gravity. Alternatively, as a new exotic matter, we add it to AdS gravity and study its effects on the dual boundary theory. In this paper, we systematically studied the fermionic spectrum dual to the charged quintessenceAdS black brane. The effect of quintessence was worked out and discussed. The main findings are summarized as follows.

- The fermionic system dual to quintessence-AdS black brane exhibits non-Fermi liquid behavior. The introduction of quintessence aggravates the degree of deviation from a Fermi liquid.

- When the dipole coupling term is introduced, this fermionic system also exhibits the phase transition from (non-)Fermi liquid to Mott phase. In particular, an interesting phenomenon is observed that the ratio $\Delta / T_{\star}$ is 
Table 3 The ratio between the width of gap and the critical temperature where the gap closes for $p=6$

\begin{tabular}{llll}
\hline $\mathrm{W}$ & -0.95 & -0.65 & -0.35 \\
\hline$\Delta / T_{\star}$ & 23.45 & 23.19 & 23.11
\end{tabular}

beyond that in holographic fermionic system dual to the RN-AdS black brane, in which $\Delta / T_{\star} \simeq 10$. It is even beyond $\Delta / T_{\star} \simeq 20$, which was observed in the material of $\mathrm{VO}_{2}$. It means that when the exotic matter, quintessence, in the universe is introduced into AdS bulk spacetimes, it also plays an important role and can model some new phenomena in the dual boundary field theory.

It was proposed in [56] that by adding an alternative Lorentz violating boundary term into the fermionic action, one can explore the holographic non-relativistic fixed point and a flat band of gapless excitation of the Green function was observed. More interesting features on the holographic nonrelativistic fermionic spectrum have been present in $[24,29$ $31,38,57,58]$. So it would be very interesting to employ the Lorentz violating boundary term instead of the standard boundary condition in our model and study the holographic non-relativistic fixed point dual to the quintessence AdS black brane. We shall publish the results elsewhere in the near future.

Acknowledgements This work is supported by the Natural Science Foundation of China under Grant nos. 11705161, 11775036 and 11305018. X. M. Kuang is also supported by Natural Science Foundation of Jiangsu Province under Grant no. BK20170481 and she is also funded by Chilean FONDECYT Grant no.3150006. J. P. Wu is also supported by Natural Science Foundation of Liaoning Province under Grant no. 201602013.

Open Access This article is distributed under the terms of the Creative Commons Attribution 4.0 International License (http://creativecomm ons.org/licenses/by/4.0/), which permits unrestricted use, distribution, and reproduction in any medium, provided you give appropriate credit to the original author(s) and the source, provide a link to the Creative Commons license, and indicate if changes were made. Funded by SCOAP ${ }^{3}$.

\section{References}

1. J.M. Maldacena, The large $\mathrm{N}$ limit of superconformal field theories and supergravity. Adv. Theor. Math. Phys. 2, 231 (1998)

2. J.M. Maldacena, The large $\mathrm{N}$ limit of superconformal field theories and supergravity. Int. J. Theor. Phys. 38, 1113 (1999)

3. S.S. Gubser, I.R. Klebanov, A.M. Polyakov, A semiclassical limit of the gauge string correspondence. Nucl. Phys. B 636, 99 (2002)

4. E. Witten, Anti-de Sitter space and holography. Adv. Theor. Math. Phys. 2, 253 (1998)

5. S.S. Lee, A non-Fermi liquid from a charged black hole: a critical Fermi ball. Phys. Rev. D 79, 086006 (2009). arXiv:0809.3402
6. H. Liu, J. McGreevy, D. Vegh, Non-Fermi liquids from holography. Phys. Rev. D 83, 065029 (2011). arXiv:0903.2477

7. M. Cubrovic, J. Zaanen, K. Schalm, String theory, quantum phase transitions and the emergent Fermi-liquid. Science 325, 439-444 (2009). arXiv: 0904.1993

8. T. Faulkner, H. Liu, J. McGreevy, D. Vegh, Emergent quantum criticality, Fermi surfaces and AdS $_{2}$. Phys. Rev. D 83, 125002 (2011). arXiv:0907.2694

9. S.A. Hartnoll, J. Polchinski, E. Silverstein, D. Tong, Towards strange metallic holography. JHEP 04, 120 (2010). arXiv:0912.1061

10. M.M. Wolf, Violation of the entropic area law for Fermions. Phys. Rev. Lett. 96, 010404 (2006). arXiv:quant-ph/0503219

11. B. Swingle, Entanglement entropy and the Fermi surface. Phys. Rev. Lett. 105, 050502 (2010). arXiv:0908.1724

12. J.P. Wu, Holographic fermions in charged Gauss-Bonnet black hole. JHEP 07, 106 (2011). arXiv:1103.3982 [hep-th]

13. N. Ogawa, T. Takayanagi, T. Ugajin, Holographic Fermi surfaces and entanglement entropy. JHEP 1201, 125 (2012). arXiv: 1111.1023

14. L. Huijse, S. Sachdev, B. Swingle, Hidden Fermi surfaces in compressible states of gauge-gravity duality. Phys. Rev. B 85, 035121 (2012). arXiv:1112.0573 [cond-mat.str-el]

15. U. Gursoy, E. Plauschinn, H. Stoof, S. Vandoren, Holography and ARPES sum-rules. JHEP 1205, 018 (2012). arXiv: 1112.5074

16. J.P. Wu, Some properties of the holographic fermions in an extremal charged dilatonic black hole. Phys. Rev. D 84, 064008 (2011). arXiv:1108.6134 [hep-th]

17. J.P. Wu, Holographic fermions on a charged Lifshitz background from Einstein-Dilaton-Maxwell model. JHEP 1303, 083 (2013)

18. M. Alishahiha, M.R. Mohammadi Mozaffar, A. Mollabashi, Fermions on Lifshitz background. Phys. Rev. D 86, 026002 (2012). arXiv:1201.1764 [hep-th]

19. L.Q. Fang, X.H. Ge, X.M. Kuang, Holographic fermions in charged Lifshitz theory. Phys. Rev. D 86, 105037 (2012). arXiv:1201.3832 [hep-th]

20. L.Q. Fang, X.H. Ge, J.P. Wu, H.Q. Leng, Anisotropic Fermi surface from holography. Phys. Rev. D 91, 126009 (2015). arXiv: 1409.6062 [hep-th]

21. L.Q. Fang, X.M. Kuang, Holographic fermions in anisotropic Einstein-Maxwell-Dilaton-Axion theory. Adv. High Energy Phys. 2015, 658607 (2015)

22. Z. Fan, Holographic fermions in asymptotically scaling geometries with hyperscaling violatio. Phys. Rev. D 88, 026018 (2013). arXiv:1303.6053 [hep-th]

23. Z. Fan, Dynamic Mott gap from holographic fermions in geometries with hyperscaling violation. JHEP 08, 119 (2013). arXiv:1305.1151 [hep-th]

24. X.-M. Kuang, E. Papantonopoulos, B. Wang, J.-P. Wu, Formation of Fermi surfaces and the appearance of liquid phases in holographic theories with hyperscaling violation. arXiv:1409.2945

25. C.J. Luo, X.M. Kuang, F.W. Shu, Charged Lifshitz black hole and probed Lorentz-violation fermions from holography. Phys. Lett. B 769, 7 (2017). arXiv:1612.01247 [hep-th]

26. M. Edalati, R.G. Leigh, P.W. Phillips, Dynamically generated Mott gap from holography. Phys. Rev. Lett. 106, 091602 (2011). arXiv: 1010.3238

27. M. Edalati, R.G. Leigh, K.W. Lo, P.W. Phillips, Dynamical gap and cuprate-like physics from holography. Phys. Rev. D 83, 046012 (2011). arXiv:1012.3751

28. D. Guarrera, J. McGreevy, Holographic Fermi surfaces and bulk dipole couplings. arXiv: 1102.3908

29. J.P. Wu, The charged Lifshitz black brane geometry and the bulk dipole coupling. Phys. Lett. B 728, 450 (2014)

30. J.P. Wu, Emergence of gap from holographic fermions on charged Lifshitz background. JHEP 1304, 073 (2013) 
31. J.P. Wu, Holographic fermionic spectrum from Born-Infeld AdS black hole. Phys. Lett. B 758, 440 (2016). arXiv:1705.06707 [hepth]

32. X.M. Kuang, B. Wang, J.P. Wu, Dipole coupling effect of holographic fermion in the background of charged Gauss-Bonnet AdS black hole. JHEP 07, 125 (2012). arXiv:1205.6674

33. X.M. Kuang, B. Wang, J.P. Wu, Dynamical gap from holography in the charged dilaton black hole. Class. Quantum Gravity 30, 145011 (2013). arXiv:1210.5735 [hep-th]

34. X.M. Kuang, E. Papantonopoulos, B. Wang, J.P. Wu, Dynamically generated gap from holography in the charged black brane with hyperscaling violation. JHEP 1504, 137 (2015), arXiv:1411.5627 [hep-th]

35. L.Q. Fang, X.H. Ge, X.M. Kuang, Holographic fermions with running chemical potential and dipole coupling. Nucl. Phys. B 877, 807-824 (2013). arXiv:1304.7431

36. J. Alsup, E. Papantonopoulos, G. Siopsis, K. Yeter, Duality between zeroes and poles in holographic systems with massless fermions and a dipole coupling. arXiv: 1404.4010

37. G. Vanacore, P. W. Phillips, Minding the gap in holographic models of interacting fermions. arXiv: 1405.1041

38. L.Q. Fang, X.M. Kuang, J.P. Wu, The holographic fermions dual to massive gravity. Sci. China Phys. Mech. Astron. 59(10), 100411 (2016)

39. S.A. Hartnoll, D.M. Hofman, Locally critical resistivities from Umklapp scattering. Phys. Rev. Lett. 108, 241601 (2012). arXiv:1201.3917 [hep-th]

40. Y. Liu, K. Schalm, Y.W. Sun, J. Zaanen, Lattice potentials and fermions in holographic non Fermi-liquids: hybridizing local quantum criticality. JHEP 1210, 036 (2012). arXiv:1205.5227 [hep-th]

41. Y. Ling, C. Niu, J.P. Wu, Z.Y. Xian, H. Zhang, Holographic fermionic liquid with lattices. JHEP 07, 045 (2013). arXiv:1304.2128 [hep-th]

42. Y. Ling, P. Liu, C. Niu, J.P. Wu, Z.Y. Xian, Holographic fermionic system with dipole coupling on Q-lattice. JHEP 12, 149 (2014). arXiv:1410.7323 [hep-th]

43. L.Q. Fang, X.M. Kuang, B. Wang, J.P. Wu, Fermionic phase transition induced by the effective impurity in holography. JHEP 11, 134 (2015). arXiv:1507.03121 [hep-th]

44. R. Cardenas, G. Tame, L. Yoelsy, M. Osmel, I. Quiros, Model of the universe including dark energy accounted for by both a quintessence field and a (negative) cosmological constant. Phys. Rev. D 67, 083501 (2003)
45. V. Sahni, L.M. Wang, A New cosmological model of quintessence and dark matter. Phys. Rev. D 62, 103517 (2000). arXiv:astro-ph/9910097

46. S.Y.Zhou, A new approach to quintessence and solution of multiple attractors. Phys. Lett. B 660, 7 (2008). arXiv:0705.1577 [astro-ph]

47. M. Kunz, D. Sapone, Crossing the phantom divide. Phys. Rev. D 74, 123503 (2006). arXiv:astro-ph/0609040

48. R.J. Scherrer, Purely kinetic k-essence as unified dark matter. Phys. Rev. Lett. 93, 011301 (2004). arXiv:astro-ph/0402316

49. V.V. Kiselev, Quintessence and black holes. Class. Quantum Gravity 20, 1187 (2003). arXiv:gr-qc/0210040

50. S. Chen, J. Jing, Quasinormal modes of a black hole surrounded by quintessence. Class. Quantum Gravity 22, 4651 (2005). arXiv:gr-qc/0511085

51. S. Chen, B. Wang, R. Su, Hawking radiation in a $d$ dimensional static spherically-symmetric black Hole surrounded by quintessence. Phys. Rev. D 77, 124011 (2008). arXiv:0801.2053 [gr-qc]

52. S. Chen, Q. Pan, J. Jing, Holographic superconductors in quintessence AdS black hole spacetime. Class. Quantum Gravity 30, 145001 (2013). arXiv:1206.2069 [gr-qc]

53. S. Chen, Q. Pan, J. Jing, Holographic p-wave superconductors in quintessence AdS black hole spacetime. Commun. Theor. Phys. 60, 471 (2013). arXiv: 1206.5462 [gr-qc]

54. A. Zylbersztejn, N.F. Mott, Metal-insulator transition in vanadium dioxide. Phys. Rev. B 11, 4383 (1975)

55. T. Giamarchi, Mott transition in one dimension. Physica B 230232, 975 (1997)

56. J.N. Laia, D. Tong, A holographic flat band. JHEP 11, 125 (2011). arXiv:1108.1381 [hep-th]

57. W.J. Li, H. Zhang, Holographic non-relativistic fermionic fixed point and bulk dipole coupling. JHEP 11, 018 (2011). arXiv: 1110.4559 [hep-th]

58. W.J. Li, R. Meyer, H. Zhang, Holographic non-relativistic fermionic fixed point by the charged dilatonic black hole. JHEP 01, 153 (2012). arXiv:1111.3783 [hep-th] 